\title{
Histopathological Analysis of Endometrial Biopsy in Gandaki Medical College Teaching Hospital, Pokhara, Nepal
}

\author{
Parajuli $\mathbf{B}^{1 *}$, Pun $\mathbf{G}^{1}$, Ranabhat $\mathbf{S}^{1}$, Poudel $\mathbf{S}^{1}$ \\ 'Lecturer, Department of Pathology, \\ Gandaki Medical College \& Teaching Hospital, Pokhara, Nepal
}

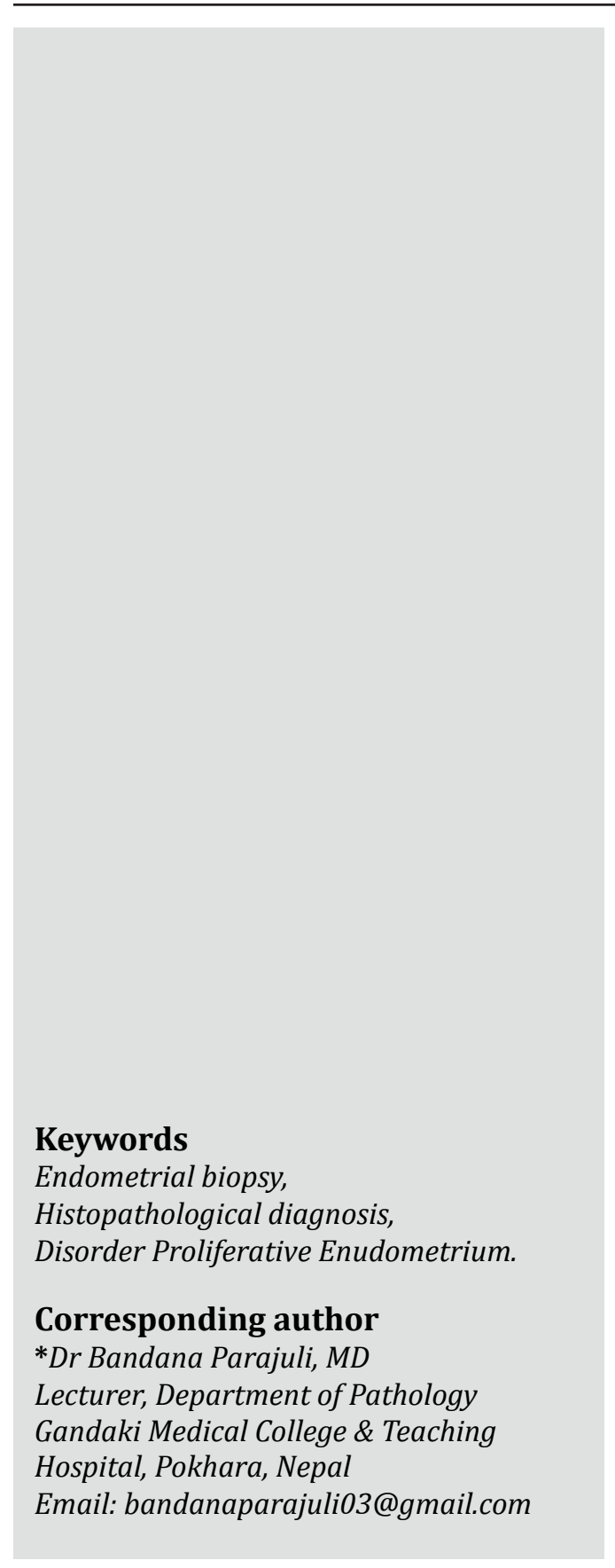

\begin{abstract}
Objective: To study the spectrum of histopathological diagnosis of endometrial lesions and their distribution according to age.

Methods: All the endometrium samples obtained by the procedure of dilatation and curettage and hysterectomy sent for histopathological examination at Pathology Department of Gandaki Medical College Teaching Hospital, Pokhara, Nepal. The study duration was total 12 months ranging from July 2016 to June 2017. All the endometrial samples were processed, sectioned at $4-6 \mu \mathrm{m}$ and stained with routine $\mathrm{H} \& \mathrm{E}$ stain. Patient's data including age, sex, procedure of the biopsy taken and histopathological diagnosis were noted. A pathologist, using Olympus microscope, reported the slides. Cases were reviewed by a second pathologist whenever necessary.
\end{abstract}

Results: A total of 128 cases were studied. The most common histopathological diagnosis was proliferative endometrium (28.9\%) followed by disorder proliferative endometrium (15.65\%). Most of the patients were in age group 36 - 45 years comprising $32.03 \%$. Hydatidiform mole comprised of $7.03 \%$ and among Hydatidiform mole, partial mole was more common. Dilatation and curettage (82.8\%) was the common procedure in compare to hysterectomy for the evaluation of endometrial lesions.

Conclusions: In this study, we observed a variety of endometrial lesions. Most of them are benign; among benign, proliferative endometrium was the common histopathological diagnosis followed by disorder proliferative endometrium. Most common presenting age group was found to be at 36 - 45 years. In evaluation of hydatidiform mole, partial mole was more frequent in compare to complete mole. Conventional dilatation and curettage is the preferred method in developing countries with limited resource to screen endometrial lesion and therefore biopsy should be sent for histopathological examination. Thus histopathological examination of routinely stained hematoxylin and eosin is readily available and widely accepted standard technique for evaluation of the endometrial lesions.

\section{INTRODUCTION}

Endometrial biopsy is routinely done test to rule out various endometrial pathology. It is easily available, safe and cheap diagnostic test. Endometrial biopsy is performed 
in cases of infertility, abnormal uterine bleeding, to rule out malignancies along with to know the phase of endometrium. However the histopathological diagnosis is highly affected by various factors like clinical history, menstrual history, age and status of use of exogenous hormones like tamoxifen.

Endometrial biopsy is done by dilatation of the cervix and curetting the endometrial cavity. If properly performed, this method can sample almost all the cavity except the cornu. However in practice, difficulties may occur during the procedure as being a blind technique done without any visual guidance and poor patient compliance. On the other side, various problems are also encountered during histopathological reporting like assessing the adequacy in scant tissue and in interpreting the artefactual changes.

Data collected from various parts of the Nepal showed the predominant histopathological diagnosis of endometrial lesion was cycling endometrium. The commonest pathology irrespective of the age group was disordered proliferative pattern and other were complications of pregnancy, benign endometrial polyp, endometrial hyperplasia, carcinoma and chronic endometritis.

This study was done to evaluate spectrum of histomorphology of the endometrial biopsy sent at Gandaki Medical College Teaching Hospital, Pokhara, Nepal.

\section{METHODS}

\section{Source of data}

All the endometrium samples received in the Department of Pathology from the patients who underwent minor and major surgery (i.e. dilatation and curettage and hysterectomy) in Gandaki Medical College Teaching Hospital, Pokhara, Nepal.

A cross sectional study was conducted in Gandaki Medical College Teaching Hospital, Pokhara, Nepal. The study duration was 12 months, from 2016, July - 2017, June. A total of 128 patients were enrolled in the study.

The endometrial biopsy specimens were received in $10 \%$ formalin. The tissues were processed and sectioned at $4-6 \mu \mathrm{m}$ of thickness using semi-automated rotary microtome. These sections were adhered to a $76 \times 25$ $\mathrm{mm}$ glass slide using egg albumin and stained with routine H\& E stain. A pathologist of the Gandaki Medical College Teaching Hospital, Pokhara, Nepal, using Olympus CX23/CX41 microscope, reported the slides. Cases were reviewed by a second pathologist whenever necessary. All the data obtained was entered in the Microsoft excel and study variables were statistically analyzed by "Statistical Package for the Social Sciences" (SPSS) 16.0. The data were expressed in terms of frequency and results were expressed in bar diagram, pie chart and tables.

\section{Inclusion criteria}

1. All the endometrial sample received in Pathology Department of Gandaki Medical College Teaching Hospital, Pokhara, Nepal.

\section{Exclusion criteria}

1. Non-compliance of the patient

2. Autolysed specimen

3. Tiny inadequate specimen difficult to process

4. Specimen not well labeled

\section{RESULTS}

A total of 128 patients whose endometrial biopsies were received in the Department of Pathology during the time interval of July 2016 to June 2017 were enrolled in this study.

Fig 1: Frequency of histopathological diagnosis $(\mathrm{N}=128)$

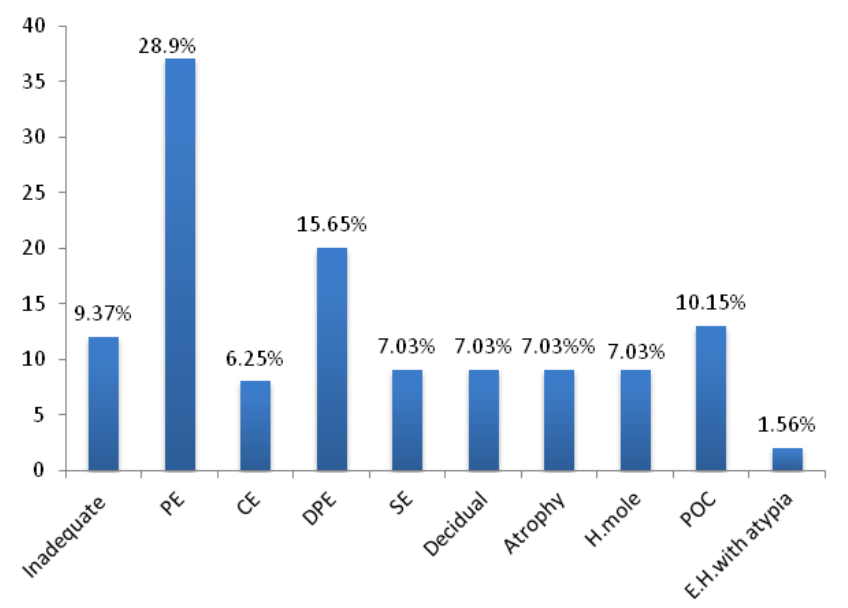

PE: Proliferative endometrium, CE: Chronic endometritis, DPE: Disorder proliferative endometrium, SE: Secretory endometrium, H. mole: Hydatidiform mole, POC: Product of conception, E.H. with atypia: Endometrial hyperplasia with atypia

In the present study, the most common histopathological diagnosis was proliferative endometrium 37 (28.9\%) followed by disorder proliferative endometrium 20 
(15.65\%) and Product of conception 13 (10.15\%), (Fig 1).

Table 1: Age wise distribution of diagnosis $(\mathrm{N}=128)$

\begin{tabular}{|c|c|c|c|c|c|c|c|}
\hline \multirow{2}{*}{ Diagnosis } & \multicolumn{6}{|c|}{ Age group in years } & \multirow{2}{*}{ Total } \\
\hline & $15-25$ & $26-35$ & $36-45$ & $46-55$ & $56-65$ & $66-75$ & \\
\hline $\begin{array}{l}\text { Inade- } \\
\text { quate }\end{array}$ & $1(0.78 \%)$ & $4(3.12 \%)$ & $4(3.12 \%)$ & $1(0.78 \%)$ & $1(0.78 \%)$ & $1(0.78 \%)$ & 12 \\
\hline P.E. & 0 & $6(4.68 \%)$ & $16(12.5 \%)$ & $13(10.15 \%)$ & $1(0.78 \%)$ & $1(0.78 \%)$ & 37 \\
\hline C.E & 0 & $1(0.78 \%)$ & $4(3.12 \%)$ & $2(1.56 \%)$ & $1(0.78 \%)$ & 0 & $8(6.25 \%)$ \\
\hline D.PE. & $1(0.78 \%)$ & $2(1.56 \%)$ & $8(6.25 \%)$ & $9(7.03 \%)$ & 0 & 0 & 20 \\
\hline S.E. & 0 & $2(1.56 \%)$ & $2(1.56 \%)$ & $5(3.9 \%)$ & 0 & 0 & $9(7.03 \%)$ \\
\hline Decidual & $3(2.34 \%)$ & $2(1.56 \%)$ & $3(2.34 \%)$ & $1(0.78 \%)$ & 0 & 0 & $9(7.03 \%)$ \\
\hline Atrophic & 0 & 0 & 0 & $1(0.78 \%)$ & $4(3.12 \%)$ & $4(3.12 \%)$ & $9(7.03 \%$ \\
\hline P. mole & $2(1.56 \%)$ & $5(3.9 \%)$ & $1(0.78 \%)$ & 0 & 0 & 0 & $8(6.25 \%)$ \\
\hline C. Mole & 0 & 0 & $1(0.78 \%)$ & 0 & 0 & 0 & $1(0.78 \%)$ \\
\hline $\begin{array}{l}\text { EH. with } \\
\text { alypia }\end{array}$ & 0 & 0 & $\%)$ & $1(0.78 \%)$ & 0 & 0 & $2(1.56 \%)$ \\
\hline POC & $3(2.34 \%)$ & $9(7.03 \%)$ & $1(0.78 \%)$ & 0 & 0 & 0 & $\begin{array}{c}13 \\
(10.15 \%)\end{array}$ \\
\hline Total & $10(7.81 \%)$ & $31(24.21 \%)$ & $41(32.03 \%)$ & $33(25.78 \%)$ & $7(5.46 \%)$ & $6(4.68 \%)$ & 128 \\
\hline
\end{tabular}

PE: Proliferative endometrium, CE: Chronic endometritis, DPE: Disorder proliferative endometrium, SE: Secretory endometrium, P. mole: partial hydatidiformmole, C. mole: Complete hydatidiform mole, POC: Product of conception, E.H. with atypia: Endometrial hyperplasia with atypia.

In this study, the common histopathological finding was proliferative endometrium and the common age group was 36 - 45 years (Table 1 ).

Table 2: Distribution of histological diagnosis according to the procedure of biopsy $(\mathrm{N}=128)$

\begin{tabular}{|c|c|c|c|c|}
\hline \multirow[t]{2}{*}{ Diagnosis } & \multicolumn{3}{|c|}{ Procedure } & \multirow[t]{2}{*}{ Total } \\
\hline & DC & VH & ТАH & \\
\hline Inadequate & 12 (9.37\%) & 0 & 0 & $12(9.37 \%)$ \\
\hline $\begin{array}{l}\text { Proliferative } \\
\text { endometrium }\end{array}$ & 24 (18.75\%) & 0 & $13(10.15 \%)$ & 37 (28.9\%) \\
\hline $\begin{array}{l}\text { Chronic endo- } \\
\text { metritis }\end{array}$ & $7(5.46 \%)$ & 0 & $1(0.78 \%)$ & $8(6.25 \%)$ \\
\hline DPE & $18(14.06 \%)$ & 0 & $2(1.56 \%)$ & 20 (15.65\%) \\
\hline $\begin{array}{l}\text { Secretory } \\
\text { phase }\end{array}$ & $9(7.03 \%)$ & 0 & 0 & $9(7.03 \%)$ \\
\hline Decidual & $9(7.03 \%)$ & 0 & 0 & $9(7.03 \%)$ \\
\hline Atrophic & $3(2.34 \%)$ & $2(1.56 \%)$ & $4(3.12 \%)$ & $9(7.03 \%)$ \\
\hline P mole & $8(6.25 \%)$ & 0 & 0 & $8(6.25 \%)$ \\
\hline $\begin{array}{l}\text { E.H.with } \\
\text { atypia }\end{array}$ & $2(1.56 \%)$ & 0 & 0 & $2(1.56 \%)$ \\
\hline POC & $13(10.15 \%)$ & 0 & 0 & $13(10.15 \%)$ \\
\hline C. Mole & $1(0.78 \%)$ & 0 & 0 & $1(0.78 \%)$ \\
\hline Total & $106(82.8 \% \%)$ & $2(1.56 \%)$ & $20(15.62 \%)$ & $128(100 \%)$ \\
\hline
\end{tabular}

DPE: Disorder proliferative endometrium, SE: Secretory endometrium, P. mole: partial hydatidiformmole, C. mole: Complete hydatidiform mole, POC: Product of conception, E.H. with atypia: Endometrial hyperplasia with atypia
Current study showed, the most common procedure for endometrial sampling was dilatation and curettage 106 (82.8\%) followed by total abdominal hysterectomy 20 (15.62\%) and vaginal hysterectomy 2 (1.56\%) (Table 2).

Fig 2: Distribution of hydatidiform mole $(n=9)$

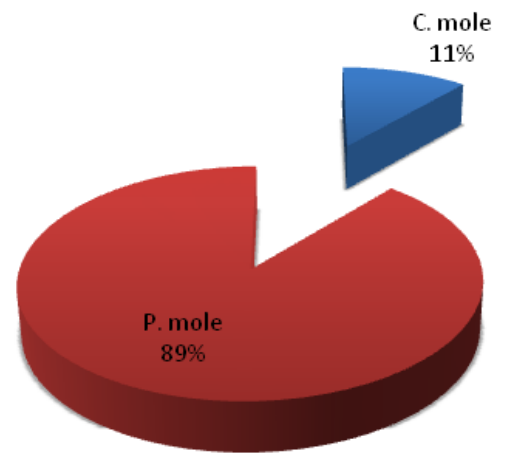

C. Mole: Complete hydatidiform mole, P. Mole: Partial hydatidiform mole

In this study, total nine hydatidiform mole were diagnosed histopathologically. Among them eight were reported as partial mole and only one was complete mole (Figure 2).

Fig 3: Chronic endometritis (H \& E, 400X)

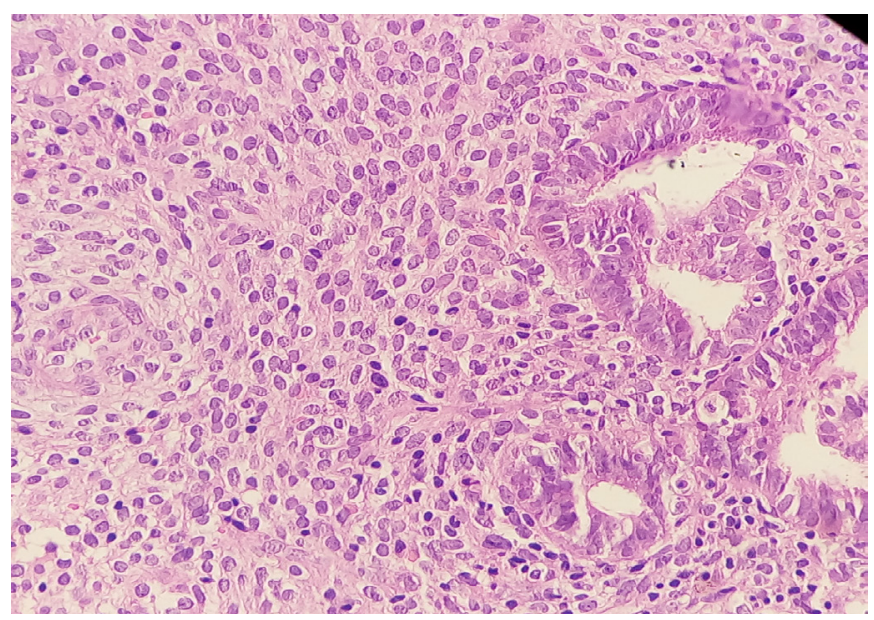

Fig 4: Partial hydatidiform mole (H \& E, 40X)

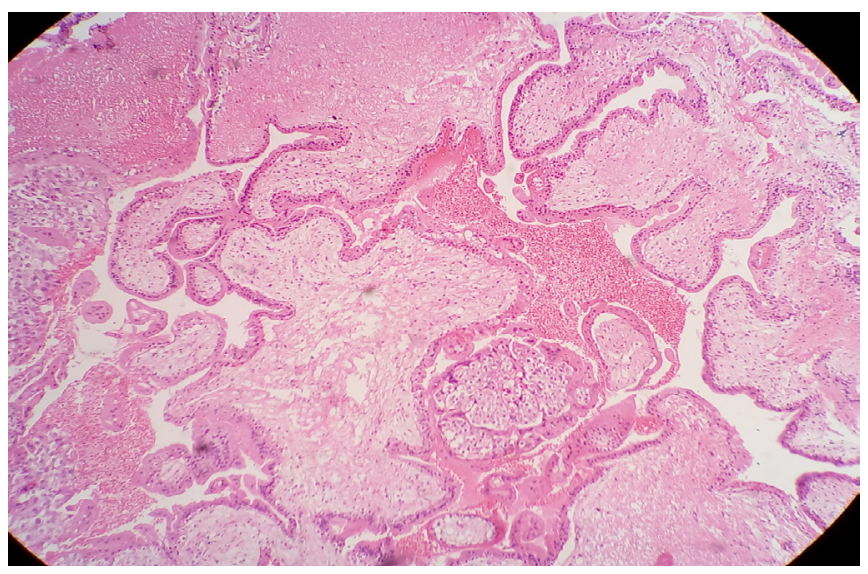


Fig 5: Spiral arterioles surrounded by predecudualized stroma (H \& E, 100x)

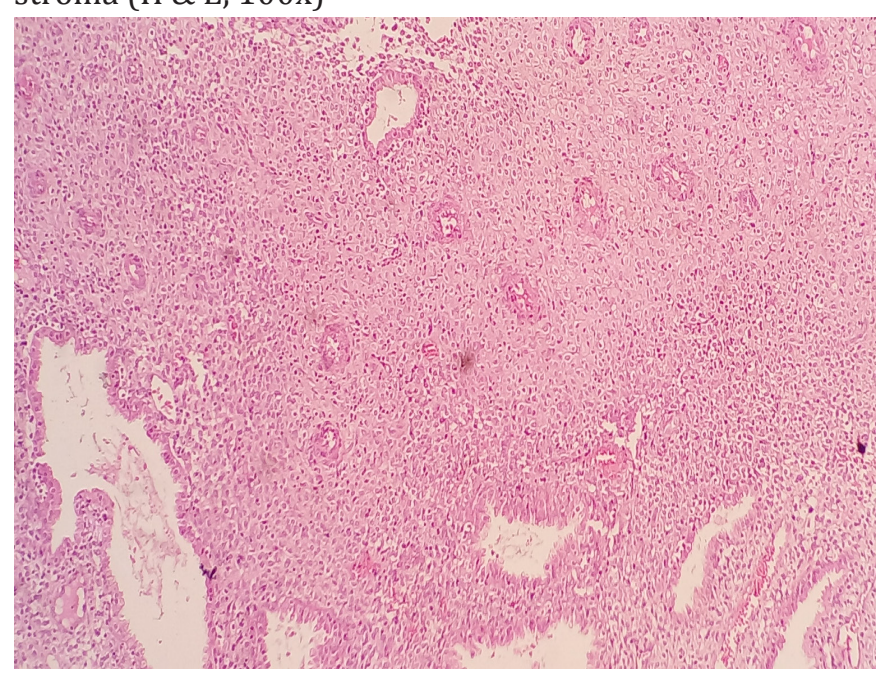

\section{DISCUSSION}

A large number of studies have suggested that endometrial biopsy is an essential step in diagnosing various endometrial pathologies. The present study was an analysis of total 128 endometrial biopsies. A spectrum of endometrial pathology was studied in relation to age and procedure of the biopsy done.

In this study, the most common age group involved was 36 - 45 years with frequency of 41 (32.03\%) followed by 26 - 35 years with frequency of 31 (24.21\%). A close finding was seen in the study done by Muzaffer et $a l^{1}$.

Like study by Nepal et $a l^{2}$, this study also showed proliferative endometrium $37(28.9 \%)$ as the most common histopathological diagnosis followed by disorder proliferative endometrium 20 (15.6\%).

Disorder proliferative endometrium was the second most common histopathological diagnosis observed in $20(15.6 \%)$ of cases and was frequent at the age group of 46 - 55 years of age which was similar to the study of Doraiswami et $a l^{3}$ and Jetley et $a l^{4}$. Several studies explained that in perimenopausal age, an anovulatory cycle are more frequent and is associated with an irregular and unpredictable pattern of bleeding. In the absence of ovulation and the production of progesterone, the endometrium responds to estrogen. Which lead to extensive proliferating endemetrium outgrows its blood supply and finally causes rupture of the glands and abnormal bleeding.

In our study, in the age group of 25 - 35 years, product of conception was the common diagnosis comprising nine $(7.03 \%)$ of cases. This can be explained by the fact that most of the women conceive at this age as being the most fertile period for reproduction. Hence, patient's presenting in this age group with abnormal uterine bleeding should be investigated for pregnancy related changes.

Atrophic endometrium was observed in nine (7.03\%) cases and all the patients were above the age of 45 years. Similar finding was seen in the study of Doraiswami et a $\beta^{3}$. However in their study, a single case of atrophic endometrium was observed in the age group of 21 - 30 years. Novak and Woodruff $^{6}$ believed that in atrophy bleeding occurs as a result of blockage of venules by overdistended glands rather than rupture of the endometrial cysts. According to Choo et $a l^{7}$, in perimenopusal age low levels of endogenous estrogen is sufficient to stimulate the endometrium to cause bleeding but do not lead to endometrial proliferation. In the study of Brunette $e t a l^{8}$, they suggested that the patients who are diagnosed as atrophic endometrium in histopathological diagnosis and are under the age of 50 years should undergo further investigations before ruling out the endometrial malignancy.

Out of total 128 cases, nine (7.03\%) were diagnosed as hydatidiform mole in the current study. But the incidence of hydatidiform mole varies greatly around the world and this is due to the lack of a clear and precise definition of the disease and over-reporting of pregnancies with gestational trophoblastic disease ${ }^{9}$.

Among hydatidiform mole, partial mole was more common than complete mole comprising eight (89\%) and one (11\%) respectively. In contrast, a study of Fukunga et al ${ }^{10}$, showed the high incidence of complete mole in compare to partial mole. The reason for low frequency of complete mole in our study may be due to the geographical variation and histological pitfalls that early complete mole is often misdiagnosed as hydropicabortus or a normal pregnancy ${ }^{11}$. In our study, five (3.9\%) patients were in the second and third decade of life, which was similar to the study done by Abdulaziz et al ${ }^{9}$. Although increased maternal age is one of the major risk factor for hydatidiform mole, only two (1.56\%) of patients were in the age group of $36-45$ years. The youngest age group observed in this study was 15 - 25 years with frequency of two (1.56\%). The low socio-economic status, early marriage and early pregnancy in our country can be considered for this rising trend in young females.

Endometrial hyperplasia with atypia was found in three 
$(1.56 \%)$ of the cases in the age group of 36 - 55 years. Jetley et $\mathrm{al}^{4}$ also found similar result in their study. In our study, we didn't found the case of endometrial hyperplasia without atypia, however in the study of Jetley et $a l^{3}$ and Baral et $a l^{12}$ the incidence of endometrial hyperplasia without atypia was common than the endometrial hyperplasia with atypia. This may be due to small number of sample in our study and lack of health awareness among female population in our society.

A total of 12 cases (9.37\%) were reported as inadequate for opinion, among them four (3.12\%) cases were found in the each age group of 26 - 35 years and 35 - 45 years, which was unlike to the study done by Nepal et $a l^{2}$. In their study, postmenopausal age was the frequent age for inadequate sampling, in which he has explained the inadequacy possibly due to the atrophic endometrium at that age. However, in this study, factor considered for inadequacy is due to the diagnostic challenges compounded by the fact that endometrial biopsies are done blindly with limited resources and randomly sampling the endometrial cavity.

The current study showed, the common procedure performed to diagnose endometrial lesion is dilatation and curettage followed by total abdominal hysterectomy and vaginal hysterectomy with frequency of 106 (82.8\%), (15.6.2\%) and two (1.56\%) respectively.

\section{CONCLUSIONS}

The present study was an attempt to know the spectrum of histopathological diagnosis of endometrial lesions and their distribution according to age. In this study, we observed a variety of endometrial lesions. Most common lesion in this study was proliferative endometrium followed by disorder proliferative endometrium, both occurring frequently in the age group of 35 - 45 years.

In total of 128 cases, only nine cases were found to be of hydatidiform mole. Among them $89 \%$ were diagnosed as partial mole and $11 \%$ as complete mole. Among neoplastic lesions, we observed a case of endometrial hyperplasia with atypia in $1.53 \%$ of the patients. However, this frequency should be validated with large scale study as the sample size is small in our study.

Among the procedure, dilatation and curettage was the preferred procedure for the histopathological examination. Hence, we conclude that histopathological examination of routinely stained hematoxylin and eosin section is widely accepted and readily available standard technique to evaluate endometrial lesions and guidance for management.

\section{REFERENCES}

1. Muzaffar M, Akhtar KA, Yasmeen S, Rehman MU, Iqbal W, Khan MA. Menstural irregularities with excessive blood loss: A clinic-pathological correlation. The Journal of Pakistan Medical Association. 2005; 55(11): 486-489.

2. Nepal et al. Histopathological analysis of endometrial biopsies in dysfunctional uterine bleeding. Journal of Pathology of Nepal. 2016; Vol 6: 910-913.

3. Doraiswami et al. Study of endometrial pathology in abnormal uterine bleeding. The Journal of Obstetrics and Gynecology of India. July-August 2011; 61(4): 426-430.

4. Jetley S, Rana S, Jairajpuri S. Morphological spectrum of endometrial pathology in middle-aged women with atypical uterine bleeding: A study of 219 cases. J Medlife Health. 2013 Oct-Dec; 4 (4): 216-220.

5. Spencer CP, Whitehead MI. Endometrial assessment revisited. Br J Obstet Gynaecol. 1999; 106: 623-32.

6. Novak Er, Woodruff JD. Novak's gynecologic and obstetric pathology with clinical and endocrine relations. $8^{\text {th }}$ ed. Philadelphia; WB Saunders publications; 1979, p185.

7. Choo YC, Mac KC, Hsuo A, Wong TS, Ma HK. Postmenopausal uterine bleeding of non-organic cause. Obstet Gynecol. 1985; 66: 225-8

8. Brunette et al. Significance of atrophy on endometrial sampling in women younger than fifty years of age. Obstetrics \& Gynecology International Journal. 2015; Vol 3 (Issue 3).

9. Abdulaziz A. Al-Mulhim. Hydatidiform mole: A study of 90 cases. Family Community Medicine. 2000 SepDec; 7(3): 57-61.

10. Fukunga $M$, Mshigome $S$, Endo $Y$. Incidence of hydatidiform moles in Tokyo Hospital: A 5 - year study (1989-1993) prospective, morphological and flow cytometric study. Hum Pathol. 1995; 26: 758- 
Original Article | Journal of Gandaki Medical College-Nepal

11. Hui P et al. Gestational trophoblastic diseases; recent advances in histopathologic diagnosis and related genetic aspects. Adv Anat Pathol. 2005; 12: 116-125.

12. Baral R, Pudasaini S. Histopathological pattern of endometrial samples in abnormal uterine bleeding. Journal of Pathology of Nepal. 2011; Vol 1: 13 - 16. 